\title{
Erfolgreiches Scheitern eines politischen Projekts
}

Rezension zu Felix Silomon-Pflug (2018): Verwaltung der unternehmerischen Stadt. Zur neoliberalen Neuordnung von Liegenschaftspolitik und -verwaltung in Berlin und Frankfurt am Main. Bielefeld: Transcript.

Abb. 1 Titelseite des Buches (Quelle: transcript Verlag)

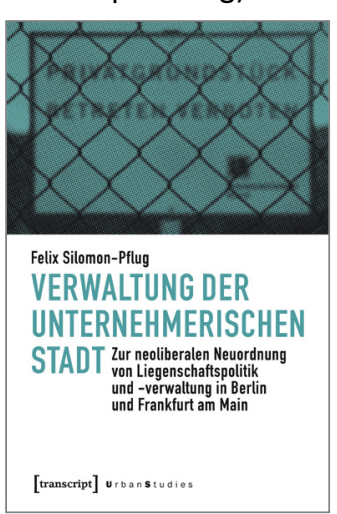

Dass in den vergangenen Jahrzehnten unter dem Label „New Public Management"Veränderungen in der Organisation und Arbeitsweise der öffentlichen Verwaltungen diskutiert und auch vollzogen wurden, davon haben wohl alle schon irgendwie gehört. Irgendetwas musste das mit gesellschaftlichen Neoliberalisierungsprozessen zu tun haben, so war lange Zeit mein Gefühl und vermutlich auch das vieler anderer Menschen. Felix Silomon-Pflug legt nun seine Dissertationsschrift Verwaltung der unternehmerischen Stadt vor, in der er sich empirisch fundiert mit diesen Entwicklungen - die in Deutschland unter dem Label „Neues Steuerungsmodell“(NSM) firmieren auseinandersetzt. Beim NSM handelt es sich um ein Anfang der 1990er Jahre durch verschiedene wissenschaftliche Akteure und Thinktanks, insbesondere der Bertelsmann Stiftung, entwickeltes Konzept. Leitbild der dort propagierten modernen Verwaltung ist das Ideal des privatwirtschaftlichen Unternehmens. Als Ziele des NSM werden Dienstleistungsorientierung, Kosteneffizienz, Effektivität und Transparenz propagiert.[1]

Silomon-Pflug stellt dem Begriff der ,Verwaltungsmodernisierung' mit seiner tendenziösen Konnotation die ,Verwaltungsneuordnung' gegenüber. Die Erkenntnisse zu dieser Neuordnung, die er aus einer vertieften Dokumentenanalyse und empirischen Fallstudien zu Berlin und Frankfurt am Main gewinnt, sind in ihrer Bedeutung und analytischen Tiefe nicht zu überschätzen. Ihre kritisch-empirische Aufarbeitung war überfällig, denn viel zu lange waren kritische Forschungsbeiträge zum Themenfeld Verwaltungsmodernisierung nur in sehr überschaubarem Maße vorhanden und hatten sich obendrein zum Teil ausschließlich den Konzepten der Reformprotagonisten[2] gewidmet (überaus überzeugend Felder 1998; 2000a; 200ob, Vogel 2006). Die Umsetzungs- oder Praxisebene blieb im Rahmen kritischer Forschung zum deutschsprachigen Raum weitgehend außen vor.[3] Für die städtische beziehungsweise kommunale Ebene stellen einzig Henrik Lebuhns (2007; 2010) Arbeiten zur sich wandelnden (nicht-)Verfügbarkeit öffentlicher Räumlichkeiten in Berlin sowie Silomon-Pflug und Susanne Heeg (2013) positive Ausnahmen dar. 


\section{Nochmal davongekommen?}

Diesen Leerstellen diametral gegenüber steht ein fast schon unüberschaubares Feld an Publikationen von grundsätzlichen Befürwortern einer Verwaltungsmodernisierung aus den Verwaltungs- und Politikwissenschaften. Diese kommen jedoch sehr einheitlich seit Mitte der 2000er Jahre zu dem Schluss, dass die Modernisierungsbemühungen trotz aller konstatierten Notwendigkeit gescheitert sind oder zumindest aus unterschiedlichen Gründen bisher keine ausreichende Wirksamkeit entfalten konnten (Bogumil 2007, Bogumil/Ebinger/Holtkamp 2011, Holtkamp 2008; 2009). Kann nun erleichtert sein, wer Schlimmes befürchtet hatte ob der geforderten und angekündigten Effizienz-, Effektivitäts- und Verschlankungsprogrammatik des NSM? Ist uns die Neoliberalisierung der Kommunalverwaltungen nochmal erspart geblieben, weil die Reformprotagonisten in Wissenschaft, Thinktanks und Verwaltung zu unfähig waren und an den Widerständen oder Eigenlogiken der selbstverwalteten Kommunalverwaltungen gescheitert sind? Keine Verbetriebswirtschaftlichung, keine Einkehr von Wettbewerb auf allen Ebenen, zwischen Kommunalverwaltungen, innerhalb von Kommunalverwaltungen, zwischen Kommunalverwaltungen und Privatwirtschaft?

\section{Erfolgreiches Scheitern...}

Wer diese Hoffnung hegt, muss sie mit jeder Seite aus Silomon-Pflugs Analyse etwas tiefer begraben. Zwar widerspricht er der These nicht, dass eine flächendeckende und umfassende Implementierung der durch das NSM vorgeschlagenen Instrumente bis heute allenfalls teilweise erfolgt ist; zwar wurden die Reformelemente oft nur teilweise umgesetzt, teilweise wieder zurückgenommen und das in sehr unterschiedlichen Geschwindigkeiten dies wird sowohl durch die Berliner als auch durch die Frankfurter Fallstudie auf je eigene Weise bestätigt - jedoch sei hierin keinesfalls ein Scheitern der Reform zu sehen. Im Gegenteil: Trotz der zeitlich wie räumlich fragmentierten Implementierung des NSM sei diese durchaus folgenreich. Dies betrifft zunächst die sehr erfolgreiche und nachhaltige Verankerung eines bestimmten Problembewusstseins in den Verwaltungsköpfen: Die Kommunalverwaltung wird - mental - zum Ort von mangelnder Effizienz und Geldverschwendung, Ort der intransparenten und permanenten Ausuferung des Staatsapparates, Ort mangelnder Innovationsfähigkeit und Kundenorientierung, so Silomon-Pflug. Dieses Problembewusstsein träfe auf die finanzielle Notlage vieler Kommunen und deute diese als intern durch Fehlsteuerung verursacht oder zumindest als vorwiegend intern lösbar und verdränge somit eine mögliche wirtschafts- und sozialpolitische Kontextualisierung der Problemlagen. Die Verbindung von materieller Not einerseits und dem skizzierten Problembewusstsein andererseits, münde in einen Prozess der permanenten Selbstüberprüfung und -optimierung von Verwaltungshandeln, der bis in die Gegenwart wirksam ist. Diese „Etablierung einer unternehmerischen Handlungs- und Organisierungsrationalität“ (Silomon-Pflug 2018: 136) gipfele in der Entwicklung immer neuer Reformprojekte, die über das ursprüngliche NSM hinausweisen (sogenanntes Strategisches Management; Human Resource Management; umfassendes Qualitätsmanagement; Public Private Partnership (PPP); in 
jüngerer Zeit systematisches Risikomanagement usw.). Aus dem einstigen Verwaltungsreformprojekt NSM, so die erste zentrale These der Arbeit, sei ein Zustand der permanenten Reform entstanden, der andauernd wirtschaftsund sozialpolitische Problemlagen als Fragen der richtigen Verwaltung reproduziere.

\section{... eines politischen Projekts}

Die Verankerung dieses Problemnarrativs und die sich daraus ergebende neue Verwaltungspraxis, so Silomon-Pflug, spielt sich jedoch nicht nur auf den Ebenen von Diskurs und Praxis ab, sondern hat höchste materielle, politisch-ökonomische Relevanz. Hierzu fokussiert Silomon-Pflug auf die materiellen Folgen der neuen Verwaltungspraxis, wofür sich das von ihm gewählte Beispiel des Umgangs mit öffentlichen Liegenschaften als besonders geeignet herausstellt. Die neuen privatwirtschaftlichen Handlungslogiken und Organisationsformen bestehen aus einer (Teil-)Abkopplung der Verwaltung von parlamentarischer Einflussnahme, Durchsetzung eines auf Wirtschaftlichkeit reduzierten Verständnisses von rationalem Handeln, Begrenzung staatlicher Aktivitäten und Eröffnung von Zugangsmöglichkeiten für privates Kapital in Bereichen einstiger staatlicher Aufgaben (Outsourcing, PPP etc.), Umschichtung staatlicher Ressourcen auf Standortpolitiken zum Beispiel durch Einrichtung privatrechtlicher Entwicklungsgesellschaften oder gezielter Infrastrukturpolitik zugunsten des Privatkapitals. Gemeinsam ist ihnen, dass der keynesianisch-wohlfahrtsstaatlich überformte Lokalstaat wieder enger an die Interessen der bürgerlichen Klassen beziehungsweise des privaten Kapitals gebunden werden kann, argumentiert Silomon-Pflug (Kap. 3.3.4). Über seine empirische Aufarbeitung der vergangenen zweieinhalb Jahrzehnte Liegenschaftspolitik in Frankfurt am Main und Berlin gelingt ihm die überzeugende Plausibilisierung der These der Verwaltungsneuordnung als politisches Projekt, die er als zweite zentrale These seiner Arbeit dem rein technisch-instrumentellen Verständnis der Mehrzahl der Reformprotagonisten entgegenstellt.

\section{Selbsttechnologien, Disziplinierung und Subjekt}

Die These des erfolgreichen Scheiterns der Verwaltungsneuordnung durch Initiierung einer zeiträumlich-fragmentierten, permanenten Reform und Selbstüberprüfung erstens sowie die These der Verwaltungsneuordnung als politisches Projekt der herrschenden Klasse zweitens, spiegeln den theoretischen Bezugsrahmen des Buches wider. Silomon-Pflug orientiert sich für die Analyse der Mikroprozesse der Neuordnung und deren polit-ökonomischer Einordnung an Michel Foucaults Gouvernementalitätstheorie sowie an der Staatstheorie Nicos Poulantzas. Die große Überzeugungskraft der oben skizzierten Kernthesen bringt die grundsätzliche Eignung und Leistungsfähigkeit dieses Analyserahmens für die untersuchten Neuordnungsprozesse zum Ausdruck. Gleichwohl hätte eine noch stärkere Orientierung der empirischen Nachzeichnungen und Analysen an den Konzepten und Kategorien dieser Theorien wahrscheinlich fruchtbar sein können. So vollzieht die Arbeit in großer Detailtiefe die Neuordnung der 
Berliner Bezirksverwaltungen nach. Diese können, auch als Ergebnis der Reform, sehr autark mit Liegenschaften umgehen, diese für ihre eigenen Zwecke einsetzen, sie aber auch ertragsorientiert beplanen, vermieten oder privatisieren, um anschließend private Räume an ihrer statt anzumieten. Diese Autonomie der Bezirksverwaltungen wird vom Berliner Senat zum Zwecke der Haushaltskonsolidierung mit Budgetierungsregeln in Interaktion gebracht, nach denen der Senat den Bezirken Gelder für bestimmte Zwecke zuweist. Die Höhe dieses Betrags orientiert sich jedoch nicht am Bedarf des jeweiligen Bezirks, sondern an den durchschnittlichen Kosten aller Bezirke. Mit einer Analyse, die noch stärker die analytischen Kategorien Foucaults nutzt, um die Interaktion von Selbsttechnologien und klassischdisziplinierender Macht ins Blickfeld zu bekommen, wäre wahrscheinlich eine noch pointiertere Nachzeichnung der Mechanismen, mit denen in Berlin ein enormer Privatisierungsdruck geschaffen wurde, möglich gewesen.

Als einer der wenigen diskutablen Punkte in Silomon-Pflugs Analyse erscheint mir die bis hierhin unerwähnt gebliebene These der Verwaltungsneuordnung als subjektloser Strategie. Hier argumentiert er mit Foucault, dass die Etablierung des Verwaltungsreformdiskurses nicht von einem strategisch-steuernden Zentrum in Form eines Subjektes ausging. Stattdessen habe es zunächst für lange Zeit keinerlei gesetzgeberische Impulse gegeben, die das Reformprogramm fest- und vorgeschrieben hätten und der angestoßene zeit-räumlich fragmentierte Neuordnungsprozess sei dezentral und aufgrund der Eigeninitiative der Kommunen erfolgt.[4] Auf der anderen Seite bestimmt Silomon-Pflug (2018:14) eine sehr überschaubare Koalition einiger Akteur*innen, zuvorderst der Bertelsmann Stiftung, der Kommunalen Gemeinschaftsstelle für Verwaltungsmanagement sowie der Hochschule für Verwaltungswissenschaften in Speyer, außerdem Personen, zuvorderst Gerhard Banner, der in allen genannten Institutionen in teils führender Funktion agierte und laut Vogel (2006) den entscheidenden Schlüsseltext (Banner 1991) zur Initiierung der Reformdebatte in Deutschland lieferte. Angesichts dieser Erkenntnisse lässt sich die These der subjektlosen Strategie hinterfragen. Silomon-Pflugs Ausführungen lassen wohl auch die Gegenthese zu, wonach hier ein klar bestimmbares Kollektivsubjekt durchaus sehr strategisch agiert und sehr wirkungsvoll einen hegemonialen Diskurs etabliert, an dem sich viele weitere Akteur*innen - bis hin zu den Gewerkschaften - in der Folge orientieren (müssen), um in der Debatte um die Zukunft der Verwaltung Gehör zu finden. So lässt sich auch der Schluss ziehen, dass es für linke Politik und kritische Stadtforschung äußerst ratsam ist, darauf zu achten, welche neuen Reformvorhaben und politischen Strategien diese Taktgeber einer permanenten Verwaltungsmodernisierung heute entwickeln und vorantreiben.

\section{Eine permanente Reform bedarf weiterer Analyse}

Insgesamt steht außer Zweifel, dass die vorliegende Arbeit einen Meilenstein für eine empirisch fundierte, kritische sozialwissenschaftliche Forschung zu Prozessen der Verwaltungsneuordnung im deutschsprachigen Raum darstellt. Die genannten (wenigen) Schwachpunkte der Arbeit können nicht darüber hinwegtäuschen, dass es Silomon-Pflug hervorragend gelingt, 
die Verwaltungsneuordnung in die Debatten um die Transformation des Städtischen und die Transformation von Staatlichkeit im Neoliberalismus einzubetten. Er zeigt die politische Dimension dieser Veränderungen überzeugend auf und motiviert dazu, sie neben den auch in dieser Zeitschrift viel diskutierten stadtpolitischen Kämpfen in politisch-strategische Überlegungen einzubeziehen. Die Arbeit macht deutlich, dass Verwaltungsneuordnung kein zeitlich begrenztes Projekt war sondern in eine permanente Transformation mündete, die auch zukünftig Gegenstand - zugegebenermaßen mühsamer - kritisch-empirischer Analyse sein muss.

\section{Endnoten}

[1] Dies soll auf der Ebene der Instrumente durch dezentrale Ressourcenverantwortung, Budgetierung, Kosten- und Leistungsrechnung, Controlling und Kontraktmanagement erreicht werden.

[2] Da eine nicht-männliche Person unter den Reformprotagonisten im deutschsprachigen Raum bisher nicht ausfindig gemacht werden konnte, steht hier bewusst nur das Maskulinum, soweit es um diesen Personenkreis geht.

[3] Eine Ausnahme stellt Pelizzari (2001) für die kantonale Ebene und das Gesundheitssystem in der Schweiz dar.

[4] Was sich gleichwohl im Laufe der 2oooer Jahre, in denen Teile der NSM-Programmatik in allen Bundesländern rechtlich verankert werden, ändert.

\section{Autor_innen}

Sofrony Riedmann ist Geograph. Sein Arbeitsschwerpunkt ist die Transformation von Staatlichkeit.

riedmann@geo.uni-frankfurt.de

Die Publikation dieses Beitrags wurde durch den Open-Access-Fonds der Goethe-Universität Frankfurt ermöglicht.

\section{Literatur}

Banner, Gerhard (1991): Von der Behörde zum Dienstleistungsunternehmen: Die Kommunen brauchen ein neues Steuerungsmodell. In: Verwaltungsführung, Organisation, Personal 13, 6-11.

Bogumil, Jörg (Hg.) (2007): Zehn Jahre neues Steuerungsmodell. Eine Bilanz kommunaler Verwaltungsmodernisierung. Berlin: Ed. Sigma.

Bogumil, Jörg / Ebinger, Falk / Holtkamp, Lars (2011): Vom Versuch, das Neue Steuerungsmodell verpflichtend einzuführen. Wirkungen des Neuen Kommunalen Finanzmanagements in NRW. In: Verwaltung \& Management 17/4, 171-180.

Felder, Michael (1998): Vom „muddling through“ zurück zum „eisernen Käfig“? Aktuelle Strategien der Verwaltungsmodernisierung. In: Z. Zeitschrift Marxistische Erneuerung 34, 91-109.

Felder, Michael (2000a): Die Transformation von Staatlichkeit. Europäisierung und Bürokratisierung in der Organisationsgesellschaft. In: Studien zur Sozialwissenschaft 213. Wiesbaden: VS.

Felder, Michael (200ob): Verwaltungsmodernisierung, die Transformation von Staatlichkeit und die neue Sozialdemokratie. In: UTOPIE kreativ 121/122, 1090-1102.

Holtkamp, Lars (2008): Das Scheitern des Neuen Steuerungsmodells. In: Der moderne Staat 2, 423-446. 
Holtkamp, Lars (2009): Governance-Konzepte in der Verwaltungswissenschaft. Neue Perspektiven auf alte Probleme von Verwaltungsreformen. In: polis 67. Hagen: Institut für Politikwissenschaft, FernUniversität Hagen.

Lebuhn, Henrik (2007): Neoliberale Technokratie und Stadtpolitik. Zur Herrschaftsfunktion von New Public Management am Beispiel Berlins. In: dérive - Zeitschrift für Stadtforschung 28, 12-16.

Lebuhn, Henrik (2010): Das Neue Steuerungsmodell und die (Markt-)Logik städtischer Verwaltungen. In: Z. Zeitschrift Marxistische Erneuerung 83, 35-45.

Pelizzari, Alessandro (2001): Die Ökonomisierung des Politischen: new public management und der neoliberale Angriff auf die öffentlichen Dienste. In: Raisons d'agir 6. Konstanz: UVK Verlagsgesellschaft.

Silomon-Pflug, Felix (2018): Verwaltung der unternehmerischen Stadt. Zur neoliberalen Neuordnung von Liegenschaftspolitik und -verwaltung in Berlin und Frankfurt am Main. Bielefeld: Transcript.

Silomon-Pflug, Felix / Heeg, Susanne (2013): Neoliberale Neuordnung städtischer Verwaltungen am Beispiel des Liegenschaftsfonds Berlin. In: Geographische Zeitschrift 101/3/4, 184-200.

Vogel, Rick(2006):Zur Institutionalisierung von New Public Management. Disziplindynamik der Verwaltungswissenschaft unter dem Einfluss ökonomischer Theorie. Wiesbaden: Deutscher Universitäts-Verlag. 